\title{
Cluster detection from surface-brightness fluctuations in SDSS data
}

\begin{abstract}
M. Bartelmann and S. D. M. White
Max-Planck-Institut für Astrophysik, PO Box 1317, 85741 Garching, Germany

Received 9 November 2001 / Accepted 4 April 2002

Abstract. Galaxy clusters can be detected as surface brightness enhancements in smoothed optical surveys. This method does not require individual galaxies to be identifiable, and enables clusters to be detected out to surprisingly high redshifts, as recently demonstrated by the Las Campanas Distant Cluster Survey (LCDCS). Here, we investigate redshift limits for cluster detection in the Sloan Digital Sky Survey (SDSS). Calibrating assumptions about the surface brightness profile, the mass-to-light ratio, and the spectral energy distribution of galaxy clusters using available observational data, we show that it should be possible to detect galaxy groups out to redshifts of $\sim 0.5$, and massive galaxy clusters out to redshifts of $\sim 1.2$ in summed $r^{\prime}+i^{\prime}+z^{\prime}$ SDSS data. Redshift estimates can be derived from the SDSS magnitudes of brightest cluster members out to redshifts near unity. Over the area of sky it covers, SDSS should find $\gtrsim 98 \%$ of the clusters detectable by the Planck satellite through the thermal Sunyaev-Zel'dovich effect. The few Planck clusters not detected in SDSS will almost all be at $z \gtrsim 1.2$.
\end{abstract}

Key words. methods: data analysis - techniques: image processing - surveys - galaxies: clusters: general cosmology: observations

\section{Introduction}

Galaxy clusters are not only interesting for studying galaxy evolution; they have also become an important tool for understanding the growth of cosmic structure and the cosmological framework in which it occurs. The construction of observed galaxy cluster samples out to redshifts unity and beyond is thus an important goal of current observational cosmology. Traditionally, galaxy clusters have been identified at optical wavelengths as regions on the sky where the number density of galaxies sufficiently exceeds its mean. This definition requires of course that the cluster galaxies be individually detectable, setting an upper limit to redshifts at which galaxy clusters can be found.

Dalcanton (1996) proposed that clusters could be detected as regions on the sky where the surface brightness exceeds the average sky brightness. Her suggested procedure consists of removing galaxies from carefully flat-fielded images, smoothing the residual image with a kernel whose width should approximately match the angular extent of galaxy clusters at intermediate redshifts, and searching for peaks in the smoothed surface-brightness distribution which sufficiently exceed the noise level of the smoothed sky background.

Gonzalez et al. (2001) successfully applied this technique to the Las Campanas Distant Cluster Survey (LCDCS) data taken with the Las Campanas Great Circle

Send offprint requests to: M. Bartelmann, e-mail: nsb@npa-garching.npg.de
Camera (Zaritsky et al. 1996) and constructed a catalog of 1073 groups and clusters. Estimated redshift limits of the catalog range from $\sim 0.3$ for groups to $\sim 0.8$ for massive galaxy clusters.

Being intrinsically highly uniform by construction, drift-scan surveys like the LCDCS provide an optimal data basis for the application of this cluster-detection technique. In this paper, we estimate the redshift limits expected for the largest ongoing drift-scan survey, the Sloan Digital Sky Survey (SDSS; York et al. 2000). Compared to the LCDCS, the SDSS uses a mirror area larger by a factor of 6.25, an exposure time shorter by a factor of 3.6, and a system of broad-band filters rather than a single, very broad filter. On the whole, the flux limit of the SDSS is expected to be roughly a factor of 1.6 below that of the LCDCS, which should allow the detection of massive clusters out to redshifts beyond unity. We study this expectation in detail in this paper. We note that cluster catalogues constructed from the SDSS by more traditional techniques (i.e. based on galaxy catalogues) are already expected to be complete to $z \sim 0.4$ (Kim et al. 2001).

Section 2 describes our assumptions. Results are presented in Sect. 3, and we summarise our conclusions in Sect. 4.

\section{Assumptions}

Throughout, we adopt a flat, low-density cosmological model with matter density parameter $\Omega_{0}=0.3$, 
Table 1. Effective wavelengths $\lambda_{\text {eff }}$, effective band widths $\Delta \lambda_{\text {eff }}$, and flux sensitivity quantities $Q$ of the five SDSS bands. Also listed is the sky brightness in the five SDSS photometric bands at the SDSS telescope site at Apache Point, and corresponding counts. The last row gives the 5- $\sigma$ Poisson fluctuation level of the sky background. $\mu_{\text {sky }}$ and $\Delta \mu_{\text {sky }}$ are given in magnitudes per square arcsec.

\begin{tabular}{lrrrrr}
\hline \hline band & $u^{\prime}$ & $g^{\prime}$ & $r^{\prime}$ & $i^{\prime}$ & $z^{\prime}$ \\
\hline$\lambda_{\text {eff }}[\AA]$ & 3546 & 4670 & 6156 & 7471 & 8918 \\
$\Delta \lambda_{\text {eff }}[\AA]$ & 457 & 928 & 812 & 893 & 1183 \\
$Q$ & 0.0171 & 0.0893 & 0.0886 & 0.0591 & 0.0099 \\
$\mu_{\text {sky }}$ & 21.8 & 21.3 & 20.5 & 19.5 & 18.3 \\
counts & 470 & 3930 & 8140 & 13630 & 6897 \\
$\Delta \mu_{\text {sky }}$ & 23.4 & 24.0 & 23.5 & 22.9 & 21.4 \\
\hline
\end{tabular}

cosmological constant corresponding to density parameter $\Omega_{\Lambda}=0.7$, and a Hubble constant $H_{0}=70 \mathrm{~km} \mathrm{~s}^{-1} \mathrm{Mpc}^{-1}$. The CDM power spectrum is taken to have primordial spectral slope $n=1$ and is normalised using $\sigma_{8}=0.93$ so as to reproduce the local abundance of galaxy clusters.

\subsection{The SDSS photometric system}

The SDSS photometric system uses five wide, almost nonoverlapping bands covering the wavelength range between $3000 \AA$ and $11000 \AA$. The recently re-measured filter response functions $S_{\nu}$ are shown in Fig. 1 (M. Strauss, private communication). The effective wavelengths $\lambda_{\text {eff }}$, effective band widths $\Delta \lambda_{\text {eff }}$, and flux sensitivity quantities

$Q=\int \mathrm{d}(\ln \nu) S_{\nu}$

of the five bands are summarised in Table 1.

The system is described in detail by Fukugita et al. (1996). Its zero points are placed on the spectrophotometric $A B$ magnitude system, so that magnitudes can directly be converted to fluxes in physical units. Given the spectral flux $F_{\nu}$ in units of erg cm $\mathrm{cm}^{-2} \mathrm{~s}^{-1} \mathrm{~Hz}^{-1}$, the broadband $A B$ magnitude is defined as

$m=-2.5 \log _{10} \frac{\int \mathrm{d}(\ln \nu) F_{\nu} S_{\nu}}{Q}-48.60$,

with $Q$ defined in Eq. (1).

\subsection{Noise estimate}

In order to assess the noise level of the sky background, we need to relate the incoming flux to the detector counts. The number of photoelectrons released by the incoming spectral flux $F_{\nu}$ is given by

$N_{\mathrm{e}}=A t h^{-1} \int \mathrm{d}(\ln \nu) F_{\nu} S_{\nu}$,

where $h$ is Planck's constant. The survey telescope area is $A=4.9 \times 10^{4} \mathrm{~cm}^{2}$, the effective exposure time per band is $t=54.1 \mathrm{~s}$.

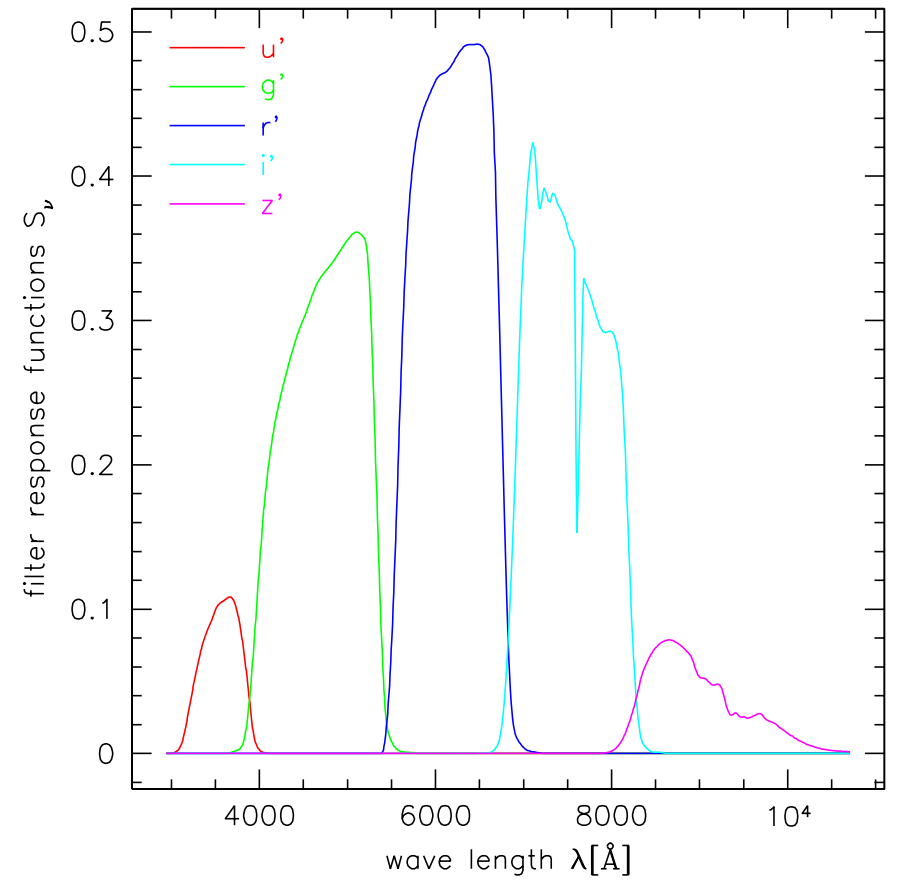

Fig. 1. Recently re-measured SDSS filter response functions. From left to right: $u^{\prime}, g^{\prime}, r^{\prime}, i^{\prime}$, and $z^{\prime}$.

The main sources of noise are the count fluctuations of the sky brightness and the brightness fluctuations in galaxies just below the SDSS detection limit. For the sky brightness $\mu_{\text {sky }}$ at the SDSS telescope site at Apache Point, we use values measured by M. Richmond (see Table 1, given in magnitudes per square arcsec). Given the area $A$ of the SDSS survey telescope, the effective exposure time $t$, and the SDSS response functions, the brightness of the night sky can be converted to counts, which are listed for each band in Table 1. The Poisson fluctuation level of the night sky per square arcsec is on the order of a few per cent. The $5-\sigma$ Poisson fluctuation in magnitudes per square arcsec is given in the last row of Table 1.

The variance of the sky brightness contributed by galaxies has two components, one from Poisson fluctuations in the galaxy number counts and one from the autocorrelation of galaxies. Following the scheme suggested by Dalcanton (1996), we assume that clusters directly detectable by counting individual galaxies have already been identified, and that galaxies brighter than the source detection threshold have been removed from the data. Noise is therefore only contributed by galaxies below the detection threshold.

The variance from Poisson fluctuations in the undetected galaxy counts in a smoothing window of solid angle $\delta \Omega$ is

$\operatorname{Var}\left(S_{\text {gal }}^{(\mathrm{P})}\right)=\delta \Omega \int_{0}^{F_{\max }} \mathrm{d} F F^{2} n(F)$,

where $n(F) \mathrm{d} F$ is the number of galaxies per unit solid angle as a function of flux $F$, and $F_{\max }$ is the flux at the detection limit. We evaluate Eq. (4) using the $I$-band 
counts measured by Woods \& Fahlman (1997), Postman et al. (1998) and Metcalfe et al. (2001), and extrapolating beyond $I=28$ with a power-law fitted to the faint end of the measured number-count distribution.

The second contribution from undetected galaxies to the variance of the sky brightness is due to the galaxy autocorrelation,

$$
\begin{aligned}
\operatorname{Var}\left(S_{\text {gal }}^{(\mathrm{C})}\right)= & \int_{0}^{F_{\max }} \mathrm{d} F_{1} \int_{0}^{F_{\max }} \mathrm{d} F_{2} F_{1} n\left(F_{1}\right) F_{2} n\left(F_{2}\right) \\
& \times \delta \Omega \int \mathrm{d}^{2} \theta W(|\boldsymbol{\theta}|) w\left(F_{1}, F_{2} ;|\boldsymbol{\theta}|\right),
\end{aligned}
$$

where $W(\theta)$ is the smoothing filter and $w\left(F_{1}, F_{2} ; \theta\right)$ is the angular correlation function of galaxies with fluxes $F_{1}$ and $F_{2}$ separated by the angle $\theta$. We use the $I$-band correlation amplitude measured by Postman et al. (1998), assume $w(\theta) \propto \theta^{-0.8}$, and conservatively replace $w\left(F_{1}, F_{2} ; \theta\right)$ by $w\left(\max \left(F_{1}, F_{2}\right) ; \theta\right)$.

If we set the flux at the detection limit, $F_{\max }$, corresponding to an $I$-band magnitude of 22 and assume a Gaussian smoothing filter $0.5^{\prime}$ wide, the combined variance from Poisson and correlated fluctuations in the counts of undetected galaxies amounts to only about $10 \%$ of the variance of the sky brightness, and even up to an $I$-band limit of 21 the galaxy counts contribute less than $30 \%$ of the sky brightness variance. This shows that undetected galaxies are not the dominant source of noise.

In practice, other sources of noise may well become more important, or even dominant, for instance residuals of sky brightness fluctuations, imperfect flat-fielding, ghosts of bright-star images and so forth. The actual level of these additional noise contributions can only be evaluated when the proposed technique will be applied to real data.

\subsection{Cluster spectra}

We describe the galaxy cluster spectrum $F_{\nu}^{\mathrm{C}}$ as a weighted superposition of an early- and a late-type galaxy spectrum, $F_{\nu}^{\mathrm{E}}$ and $F_{\nu}^{\mathrm{L}}$, respectively. The combined cluster spectrum at redshift $z$ is then written as

$$
\begin{aligned}
F_{\nu}^{\mathrm{C}}(z)= & f_{E}(z) l_{E}(z) F_{\nu(1+z)}^{\mathrm{E}} \\
& +\left[1-f_{E}(z)\right] f_{L} F_{\nu(1+z)}^{\mathrm{L}}
\end{aligned}
$$

The early-type fraction of the cluster population, $f_{E}(z)$, decreases with redshift. The results of van Dokkum et al. (2000) suggest choosing a linear decrease with redshift,

$$
f_{E}(z)=0.8-0.4 z \text {. }
$$

The mass-to-light ratio of early-type galaxies in clusters decreases with increasing redshift. For the mass-to-light ratio in the $B$ band, van Dokkum et al. (1998) find $\Delta \log \left(M / L_{B}\right) \sim-0.4 z$. Adopting this relation, we assume that early-type galaxies brighten with redshift as

$$
l_{E}(z)=10^{0.4 z} \text {. }
$$

Table 2. Mass-to-light ratios in solar units of a galaxy cluster at redshift zero in the five SDSS bands. The mass-to-light ratio is normalised to 250 in the Johnson $B$ band. For comparison, the mass-to-light ratios in the conventional Johnson bands are also shown. Due to the predominantly red galaxy population, the mass-to-light ratio decreases towards longerwavelength bands.

\begin{tabular}{lrrrrr}
\hline \hline SDSS band & $u^{\prime}$ & $g^{\prime}$ & $r^{\prime}$ & $i^{\prime}$ & $z^{\prime}$ \\
$(M / L) /\left(M_{\odot} / L_{\odot}\right)$ & 362 & 229 & 162 & 134 & 101 \\
Johnson band & $U$ & $B$ & $V$ & $R$ & $I$ \\
$(M / L) /\left(M_{\odot} / L_{\odot}\right)$ & 363 & 250 & 186 & 145 & 103 \\
\hline
\end{tabular}

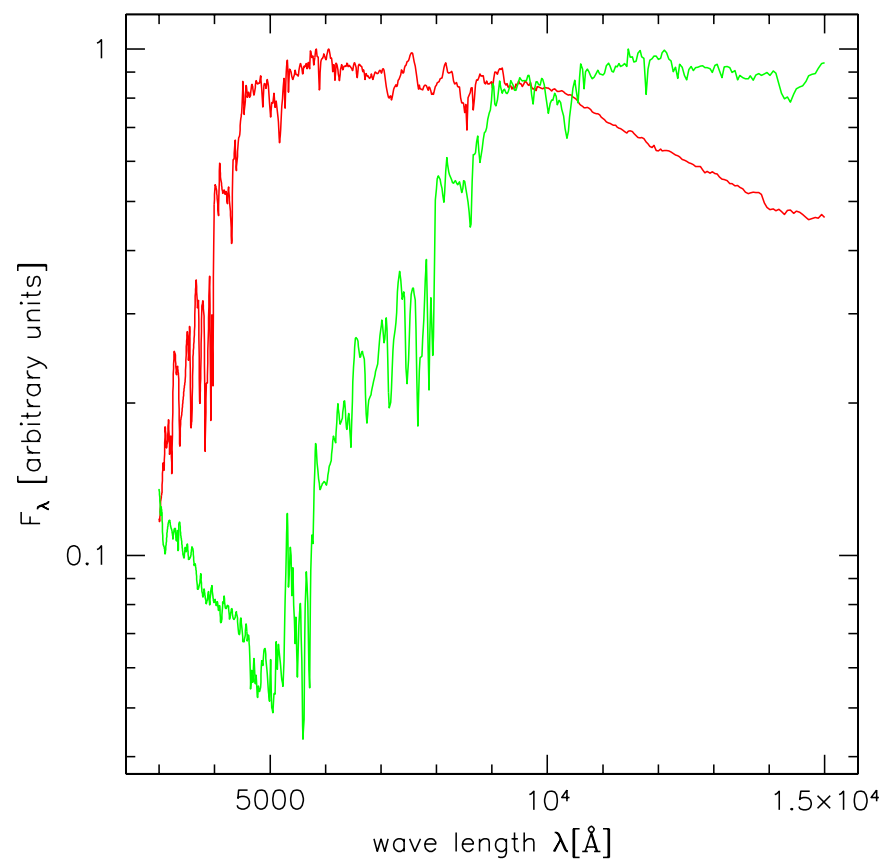

Fig. 2. Composite cluster spectra $F_{\lambda}^{\mathrm{C}}$ as functions of wavelength $\lambda$ in $\AA$, at redshifts zero (solid curve) and unity (dotted curve). Both spectra are arbitrarily normalised.

The late-type fraction increases with redshift, but we assume the mass-to-light ratio of late-type galaxies to be constant. In order to account for the fact that late-type galaxies are typically fainter than early-type galaxies, we introduce a redshift-independent factor $f_{L}<1$, which, based on the data of van Dokkum et al. (2000), we set to

$f_{L}=\frac{2}{3}$

The spectra $F_{\nu}^{\mathrm{E}, \mathrm{L}}$ themselves are redshifted, but otherwise assumed to be constant. They were kindly provided by S. Charlot (private communication).

The mass-to-light ratio of galaxy clusters is typically measured to be on the order of $M / L_{B}=250 M_{\odot} / L_{\odot}$ in the $B$ band (e.g. Bahcall et al. 1995; Carlberg et al. 1996; Carlberg et al. 1997a; Gonzalez et al. 2000), however with a scatter of $\sim 20 \%$. We normalise the combined cluster spectrum such that the $B$-band mass-to-light ratio at redshift zero is 250 in solar units. Specifically, let $F_{\nu}^{\odot}$ be the 


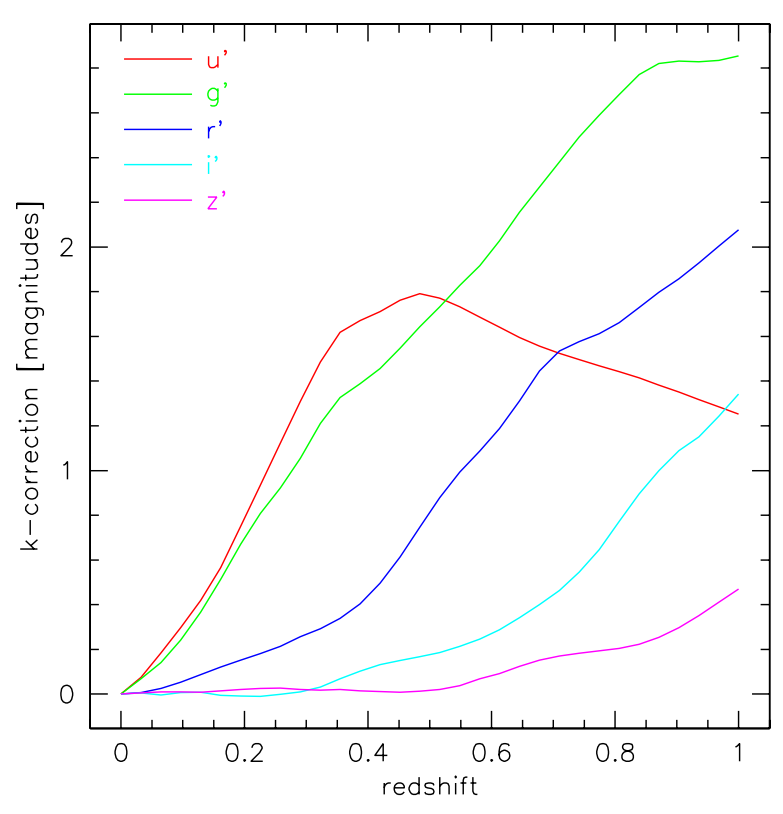

Fig. 3. Cluster $k$-corrections as a functions of redshift for $M=$ $5 \times 10^{14} M_{\odot} / h$ in the five SDSS bands (solid curve: $u^{\prime}$, dotted curve: $g^{\prime}$, short-dashed curve: $r^{\prime}$, long-dashed curve: $i^{\prime}$, dashdotted curve: $z^{\prime}$ ).

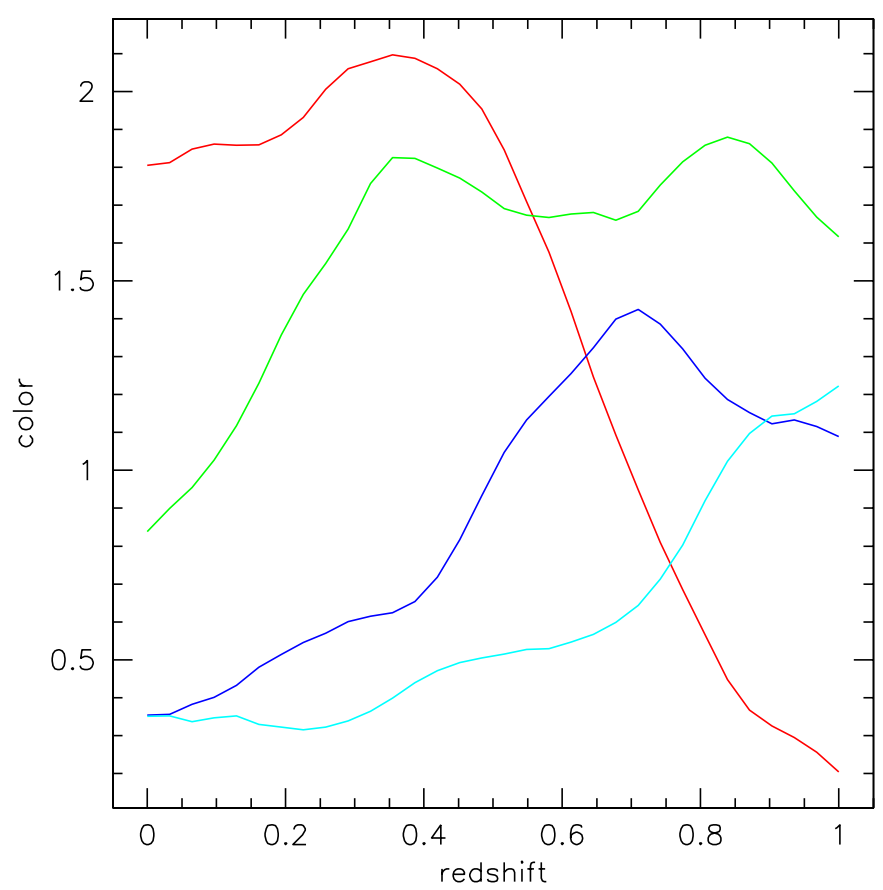

Fig. 4. Cluster colours as functions of redshift, for cluster mass $M=5 \times 10^{14} M_{\odot} / h$. Solid curve: $\left(u^{\prime}-g^{\prime}\right)$; dotted curve: $\left(g^{\prime}-r^{\prime}\right)$, short-dashed curve: $\left(r^{\prime}-i^{\prime}\right)$; long-dashed curve: $\left(i^{\prime}-z^{\prime}\right)$.

solar spectrum, then the amplitude of the cluster spectrum is chosen such that

$\int \mathrm{d}(\ln \nu) F_{\nu}^{\mathrm{C}} S_{\nu, B}=\frac{M}{250 M_{\odot}} \int \mathrm{d}(\ln \nu) F_{\nu}^{\odot} S_{\nu, B}$

is satisfied. The mass-to-ratios in all five SDSS bands and in the conventional Johnson- $U B V R I$ bands are given in Table 2.
The predominantly early-type galaxy population causes the mass-to-light ratio to decrease towards longerwavelength bands. This trend agrees well with observations; for instance, the $I$-band mass-to-light ratio of Abell 1651 was measured to be $\sim 160$ (Gonzalez et al. 2000).

Figure 2 shows arbitrarily normalised cluster spectra at redshifts zero and unity.

\subsection{Cluster k-corrections and colours}

Integrating the synthetic cluster spectrum with the SDSS filter curves as in Eq. (2), we can now compute $k$ corrections and cluster colours for the SDSS photometric system. Figure 3 displays the cluster $k$-corrections in the five SDSS bands as functions of cluster redshift.

The $k$-correction in the bluest SDSS band $\left(u^{\prime}\right)$ grows to $\sim 2.4$ mag. up to $z \sim 0.5$ and then levels off. This is because at that redshift essentially all of the cluster flux has been shifted redward of the $u^{\prime}$ filter curve, and the remaining short-wavelength part of the cluster spectrum is almost flat. The $k$ corrections in the other SDSS bands grow monotonically with redshift. Their amplitudes decrease as the filters get redder.

Figure 4 shows the four different cluster colours $\left(u^{\prime}-g^{\prime}\right),\left(g^{\prime}-r^{\prime}\right),\left(r^{\prime}-i^{\prime}\right)$ and $\left(i^{\prime}-z^{\prime}\right)$ as a function of cluster redshift.

On the whole, clusters become redder as their redshift increases, with the exception of the bluest colour shown $\left(u^{\prime}-g^{\prime}\right)$, which starts red at low redshift and then decreases beyond redshift $z \sim 0.4$ as the $3000 \AA$ break shifts through the $g^{\prime}$ band.

\subsection{Cluster surface brightness}

We assume that the average light distribution in galaxy clusters follows mass, and that the mass distribution is described by the familiar NFW density profile (Navarro et al. 1996, 1997). Data from the CNOC survey shows that this gives a very good model for the mean observed luminosity profile of rich clusters (Carlberg et al. 1997b). The three-dimensional matter density is

$\rho(x)=\frac{\rho_{\mathrm{s}}}{x(1+x)^{2}}$,

where $r_{\mathrm{s}}$ is a characteristic scale radius, $\rho_{\mathrm{s}}$ is the density scale, and $x \equiv r / r_{\mathrm{s}}$. Although two free parameters appear in (11), there is effectively only one parameter to set because the scale radius and the density scale are related. We choose the virial mass $M$ as the remaining single free parameter and compute $r_{\mathrm{s}}$ and $\rho_{\mathrm{s}}$ from it, following the prescription given in Navarro et al. (1997). The virial mass is

$M=4 \pi \rho_{\mathrm{s}} r_{\mathrm{s}}^{3}\left[\ln (1+c)-\frac{c}{1+c}\right]$,

where the concentration $c=r_{200} / r_{\mathrm{s}}$ is the ratio between the virial radius and the scale radius. 


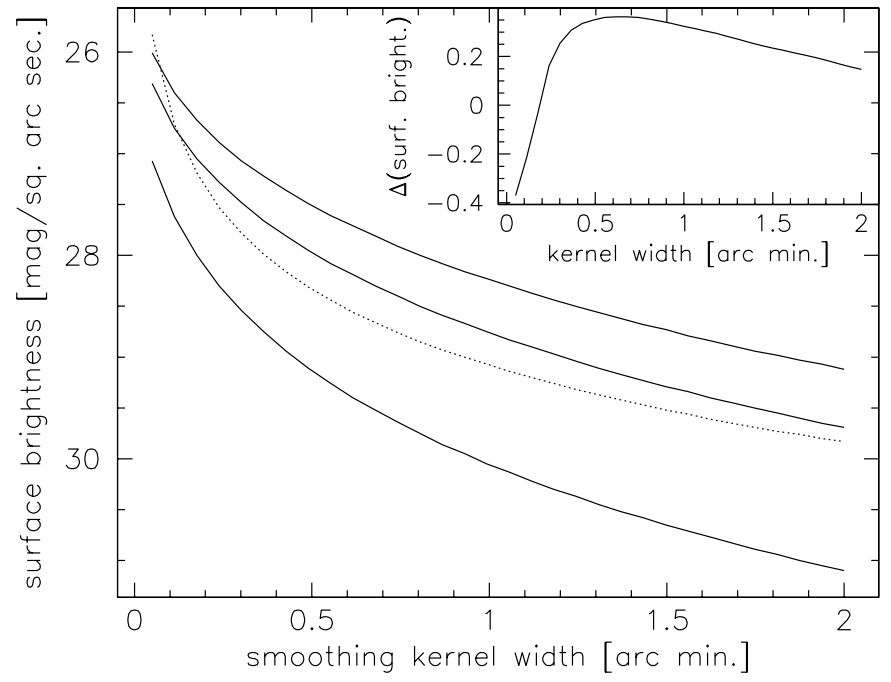

Fig. 5. Central surface brightness in the $r^{\prime}$ band (in magnitudes per square arcsec) as a function of smoothing radius for clusters of masses $M=\left(10^{14}, 3 \times 10^{14}, 10^{15}\right) M_{\odot} / h$ at redshift $z=0.75$ (solid curves from bottom to top), and the 5- $\sigma$ sky brightness fluctuations (dotted curve). The small panel shows the difference between the solid curve for $M=3 \times 10^{14} M_{\odot} / h$ and the dotted curve, i.e. the signal-to-noise ratio as a function of the smoothing radius. It has a broad peak at $\Delta \theta \sim 0.5^{\prime}$.

Given the cluster mass, its luminosity $L$ is determined by the mass-to-light ratio

$$
L=\frac{M}{(M / L)} L_{\odot} \text {. }
$$

When projected along the line of sight, its surface mass density of an NFW halo is $\Sigma(x)=\rho_{\mathrm{s}} r_{\mathrm{s}} f(x)$, with

$f(x)=\frac{2}{x^{2}-1}\left[1-\frac{2}{\sqrt{x^{2}-1}} \arctan \sqrt{\frac{x-1}{x+1}}\right]$,

(see e.g. Bartelmann 1996). Therefore, the surface brightness profile of the cluster is

$S_{\mathrm{C}}(x)=\frac{L f(x)}{4 \pi r_{\mathrm{s}}^{2}}\left[\ln (1+c)-\frac{c}{1+c}\right]^{-1} ;$

$S_{\mathrm{C}}(x)$ is the energy radiated by the cluster per unit unit surface area per unit time.

Photon conservation, and the Etherington relation between angular-diameter and luminosity distance then imply that the flux received from a cluster at redshift $z$ per unit time, frequency, detector area and solid angle is

$$
\begin{aligned}
S_{\nu}^{\mathrm{C}}(\theta)= & \frac{F_{\nu}^{\mathrm{C}}(z)}{4 \pi(1+z)^{3} 4 \pi r_{\mathrm{s}}^{2}}\left[\ln (1+c)-\frac{c}{1+c}\right]^{-1} \\
& \times f\left[\frac{D(z) \theta}{r_{\mathrm{s}}}\right]
\end{aligned}
$$

where $\theta$ is the angular separation from the projected cluster centre, $D(z)$ is the angular diameter distance to the cluster, and $F_{\nu}^{\mathrm{C}}$ is the cluster spectrum of Eq. (6), i.e. the cluster luminosity per unit frequency.

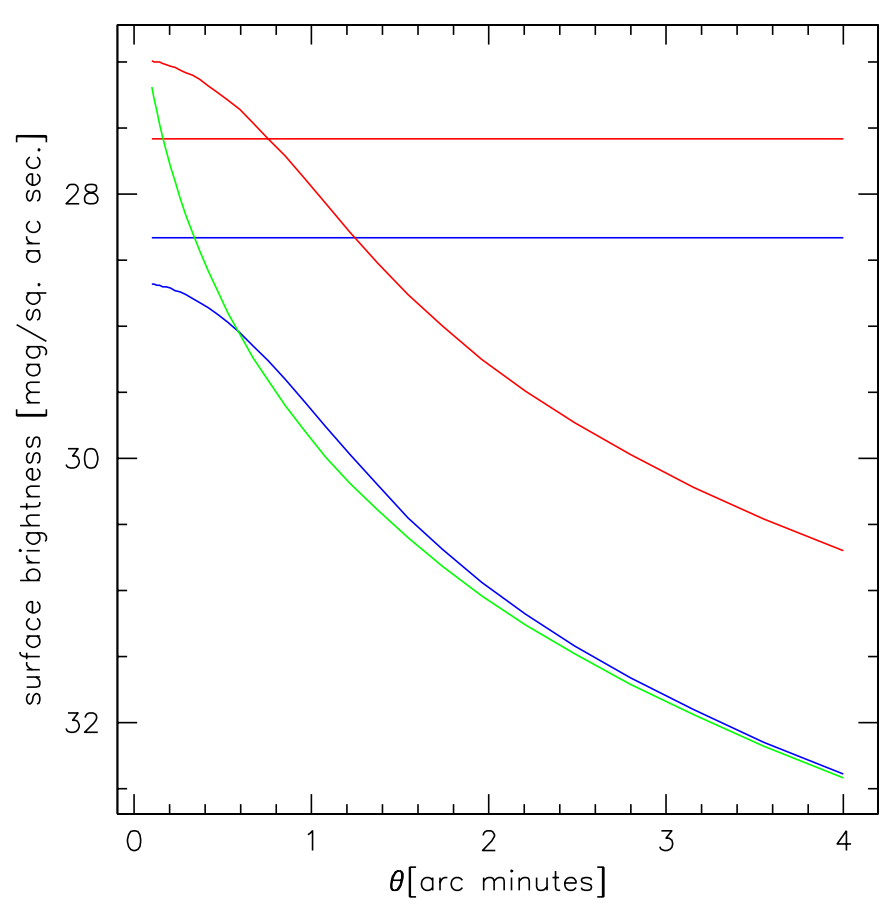

Fig. 6. Surface brightness profiles (in magnitudes per square arcsec) for a cluster of mass $M=2 \times 10^{14} M_{\odot} / h$ at redshift $z=$ 0.75 as a function of cluster-centric distance $\theta$. The assumed intrinsic NFW surface brightness profile in the $r^{\prime}$ band is shown as the dotted curve, which is smoothed by a Gaussian kernel of width $\Delta \theta=0.5^{\prime}$ (dashed curve). The solid curve shows the surface brightness summed in the $g^{\prime}, r^{\prime}$ and $i^{\prime}$ bands. The horizontal curves indicate the 5- $\sigma$ sky brightness fluctuation levels in the $r^{\prime}$ band (dashed) and the summed $g^{\prime}, r^{\prime}$ and $i^{\prime}$ bands (solid), respectively. While the smoothed cluster falls below the noise level in the $r^{\prime}$ band, it is well detected in the summed bands.

By means of Eq. $(2), S_{\nu}^{\mathrm{C}}(\theta)$ can now be converted into a surface brightness profile for the cluster in the conventional units of magnitudes per square arcsec. The dotted curve in Fig. 6 shows an example for a cluster of mass $2 \times 10^{14} M_{\odot} / h$ at redshift $z=0.75$.

\subsection{Smoothing}

The signal-to-noise ratio of a cluster detection can be increased by smoothing. We therefore convolve the projected cluster profile (16) with a Gaussian filter of width $\Delta \theta$. Exploiting the axial symmetry both of the cluster profile and of the Gaussian, and making use of the convolution theorem in Fourier space, the convolution can be transformed into

$\bar{S}_{\nu}^{\mathrm{C}}(\theta)=\int_{0}^{\infty} \mathrm{d} \phi \phi \frac{S_{\nu}^{\mathrm{C}}(\phi)}{\Delta \theta^{2}} \exp \left(-\frac{\theta^{2}+\phi^{2}}{2 \Delta \theta^{2}}\right) I_{0}\left(\frac{\theta \phi}{\Delta \theta^{2}}\right)$

where $I_{0}(x)$ is the modified Bessel function of order zero. It can be approximated by $I_{0}(x) \approx(2 \pi x)^{-1 / 2} \exp (x)$ for $x \gg 1$, in which case the convolution simplifies to

$\bar{S}_{\nu}^{\mathrm{C}}(\theta) \approx \int_{0}^{\infty} \mathrm{d} \phi \phi \frac{S_{\nu}^{\mathrm{C}}(\phi)}{\sqrt{2 \pi \theta \phi} \Delta \theta} \exp \left[-\frac{(\theta-\phi)^{2}}{2 \Delta \theta^{2}}\right]$. 


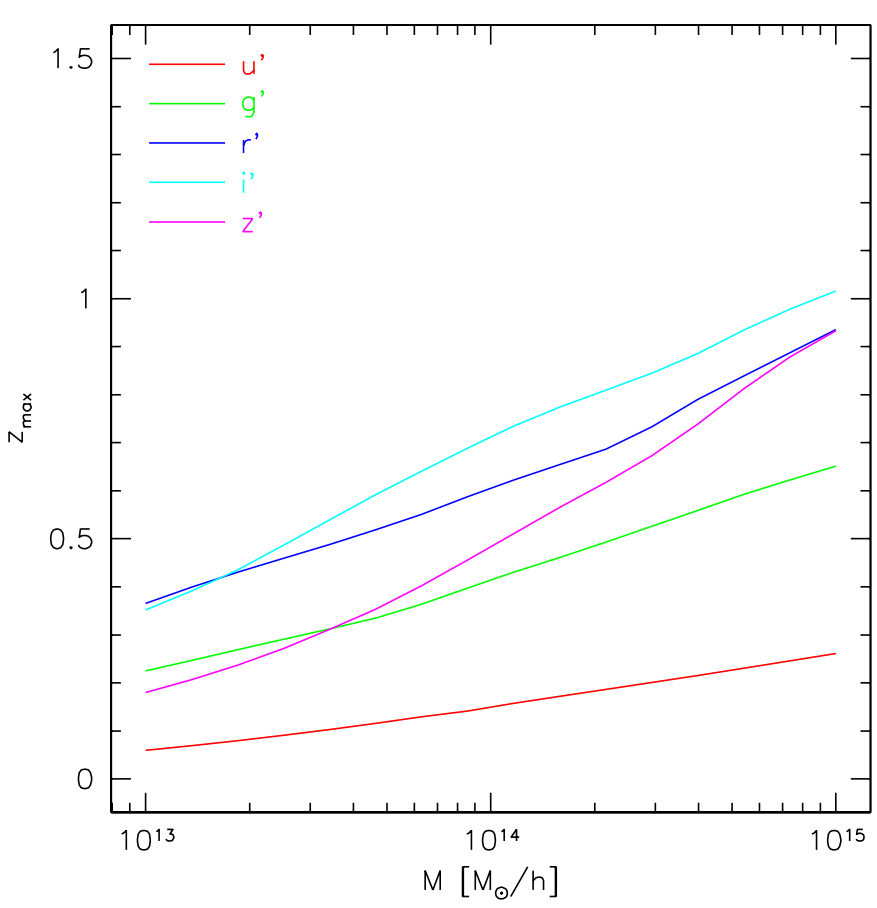

Fig. 7. 5- $\sigma$ detection limits for clusters as a function of mass in the five individual SDSS bands. Solid curve: $u^{\prime}$, dotted curve: $g^{\prime}$, short-dashed curve: $r^{\prime}$, long-dashed curve: $i^{\prime}$, dashdotted curve: $z^{\prime}$.

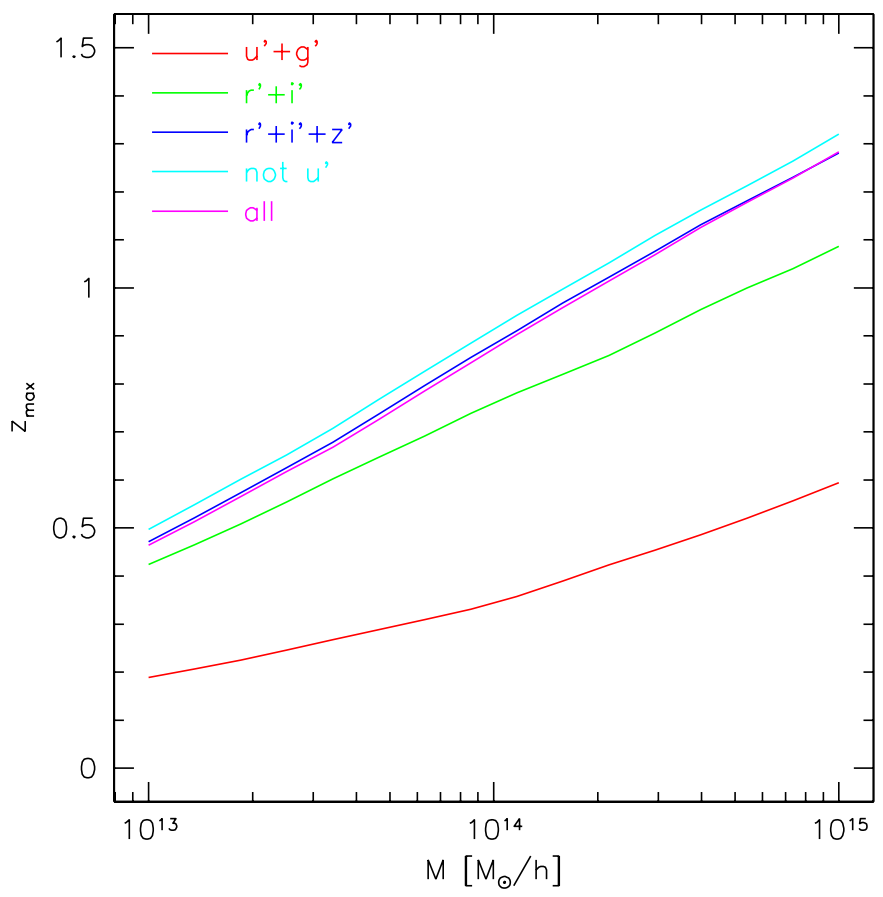

Fig. 8. 5- $\sigma$ detection limits for clusters as a function of mass in several combinations of wave bands. Solid curve: $\left(u^{\prime}+g^{\prime}\right)$; dotted curve: $\left(r^{\prime}+i^{\prime}\right)$; short-dashed curve: $\left(r^{\prime}+i^{\prime}+z^{\prime}\right)$; longdashed curve: all bands except $u^{\prime}$; dash-dotted curve: all bands.

We show in Fig. 5 the central surface brightness in the $r^{\prime}$ band of clusters with masses $M=\left(10^{14}, 3 \times\right.$ $\left.10^{14}, 10^{15}\right) M_{\odot} / h$ at redshift 0.75 , smoothed with a Gaussian kernel of varying width $\Delta \theta$, and the 5- $\sigma$ sky brightness fluctuation level. The small inserted panel shows the difference between the cluster surface brightness for $M=3 \times 10^{14} M_{\odot} / h$ and the sky noise, which indicates that the signal-to-noise ratio peaks at $\Delta \theta \sim 0.5^{\prime}$.

Figure 5 shows that the sky noise falls below the central cluster surface brightness when the smoothing kernel grows above $\approx 0.2^{\prime}$, and the height of the cluster centre above the noise reaches a broad maximum at $\Delta \theta \approx 0.5^{\prime}$. Correspondingly, we choose a smoothing kernel width of $\Delta \theta=0.5^{\prime}$ in the following.

Figure 6 illustrates the effects of smoothing and sky noise fluctuations on the surface-brightness profile of a cluster with mass $M=2 \times 10^{14} M_{\odot} / h$ at redshift $z=0.75$. The intrinsic profile is broadened by the Gaussian smoothing kernel, which has $\Delta \theta=0.5^{\prime}$. While the smoothed profile falls below the 5- $\sigma$ noise fluctuation level if data in the $r^{\prime}$ band only are used, the cluster is well detected if data in the $g^{\prime}, r^{\prime}$ and $i^{\prime}$ bands are summed.

\section{Results}

We can now proceed to compute the upper redshift limit for a significant cluster detection. We first consider individual SDSS bands. Figure 7 displays the redshift $z_{\max }$ as a function of cluster mass at which the central cluster surface brightness in each of the five SDSS bands drops below the 5- $\sigma$ limit of the sky background fluctuation, after smoothing with a Gaussian kernel of width $\Delta \theta=0.5^{\prime}$.

Clearly, the detection limit in the $u^{\prime}$ band is the poorest, reaching only out to redshift $z \sim 0.15$ at $M=$ $10^{14} M_{\odot} / h$. This is a consequence of the combined effect of the relatively high background fluctuation level, the low detector efficiency and the predominantly red cluster light. The detection limit increases rapidly with increasing filter wavelength to reach just above redshift unity in the $i^{\prime}$ band for massive clusters with $M=10^{15} M_{\odot} / h$. The relatively low $k$ correction in the $z^{\prime}$ band leads to the comparatively steep increase in the upper redshift limit despite the poor efficiency in the redmost SDSS band.

Figure 7 indicates that the detection limit can be considerably increased by summing data in several bands. Figure 8 quantifies this expectation.

The solid curve shows the detection limit for data summed in the two bluest bands $\left(u^{\prime}\right.$ and $\left.g^{\prime}\right)$, the dotted curve for data summed in the two redder bands $\left(r^{\prime}\right.$ and $i^{\prime}$ ). The increase between the two is substantial; the upper redshift limit approximately doubles. One to two tenths in redshift are gained if the $z^{\prime}$ band data are added to the $r^{\prime}$ and $i^{\prime}$ bands. Further addition of the $g^{\prime}$ band improves the limit a little more, while the relatively low signal-to-noise ratio in the $u^{\prime}$ band even lowers the detection limit if data in all bands are summed.

These results show that massive clusters with $M \sim$ $10^{15} M_{\odot} / h$ should be detectable as significant surfacebrightness enhancements in the SDSS data out to redshifts of $z \sim 1.25$ if data in the three redmost bands are summed. The redshift limit of the cluster detection increases approximately linearly with $\log _{10}\left(M h / M_{\odot}\right)$. 


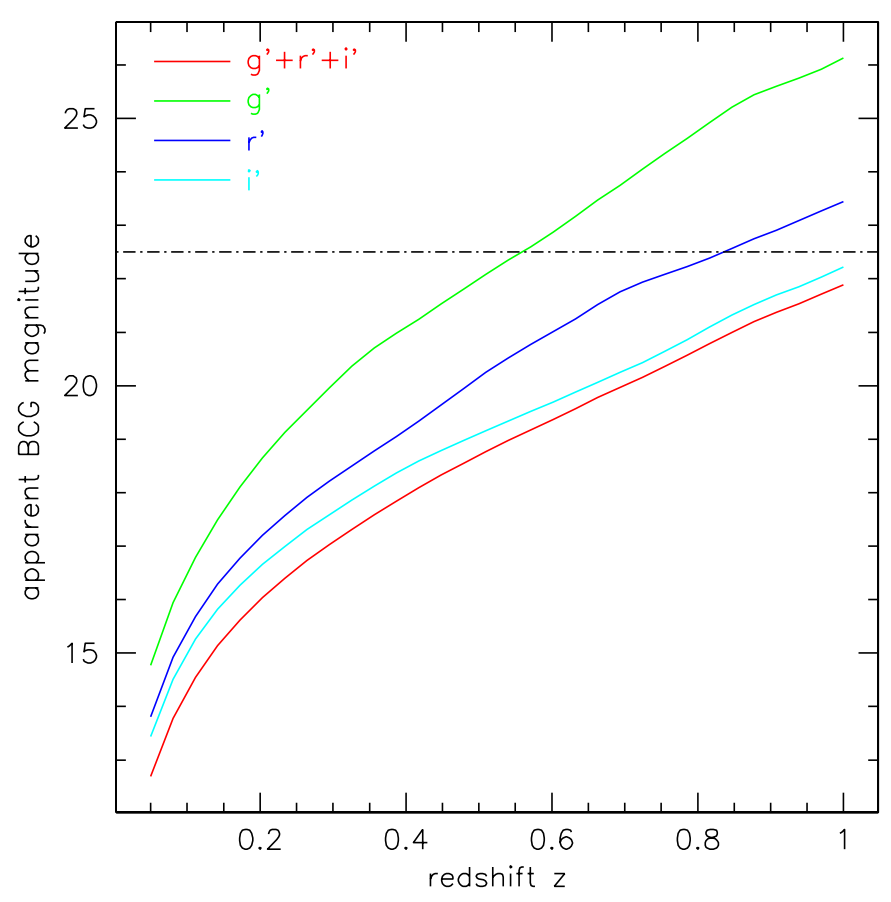

Fig. 9. Estimated magnitudes of brightest cluster galaxies in the $g^{\prime}, r^{\prime}$ and $i^{\prime}$ SDSS filter bands (dotted, short-dashed, and long-dashed curves as indicated), and in the summed filters (solid curve). The dash-dotted curve indicates a conservative detection limit of $22.5 \mathrm{mag}$ (approximately $5-\sigma$ ). Brightest cluster galaxies should be detectable in summed SDSS data out to redshifts around unity. When identified with galaxy cluster candidates, they will increase the reliability of the detection and allow photometric redshift estimates.

This redshift limit agrees with expectations raised by the Las Campanas Distant Cluster Survey (LCDCS; Zaritsky et al. 1997; Gonzalez et al. 2001). The LCDCS used a 1-m telescope with an effective exposure time of $194 \mathrm{~s}$, while the SDSS has a 2.5-m telescope and an effective exposure time of $54.1 \mathrm{~s}$. The product of telescope area and exposure time is therefore larger for the SDSS by $75 \%$. The LCDCS uses the broad $W$ filter. It covers the wavelength range between $4600 \AA$ and $7300 \AA$, which overlaps with the SDSS $r^{\prime}$ filter and major fractions of the $g^{\prime}$ and $i^{\prime}$ filters. The combined width of the $g^{\prime}+r^{\prime}+i^{\prime}+z^{\prime}$ filters is approximately $4000 \AA$, or $\sim 50 \%$ larger than the width of the $W$ filter. Furthermore, adding $z^{\prime}$ data helps because of the predominantly red colour of galaxy clusters. Adopting the same quantum efficiency for the SDSS and LCDCS CCDs, it seems reasonable to assume that the combined $g^{\prime}+r^{\prime}+i^{\prime}+z^{\prime}$ SDSS data lower the LCDCS flux limit by approximately a factor of $(1.5 \times 1.75)^{1 / 2} \approx 1.6$.

The LCDCS contains groups out to redshifts of $\sim 0.3$, and massive clusters out to redshifts of $\sim 0.8$, while we estimate respective redshift limits for SDSS to be $\sim 0.5$ and $\sim 1.3$. As Eq. (16) shows, the observed surface brightness $S_{\nu}^{\mathrm{C}}$ scales with redshift roughly as $(1+z)^{-3}$. The estimated improvement in the flux limit by $\sim 1.6$ thus leads to an expected increase in the redshift limit of

$z_{\max }^{(\mathrm{SDSS})} \sim 1.6^{1 / 3}\left(1+z_{\max }^{(\mathrm{LCDCS})}\right)-1$

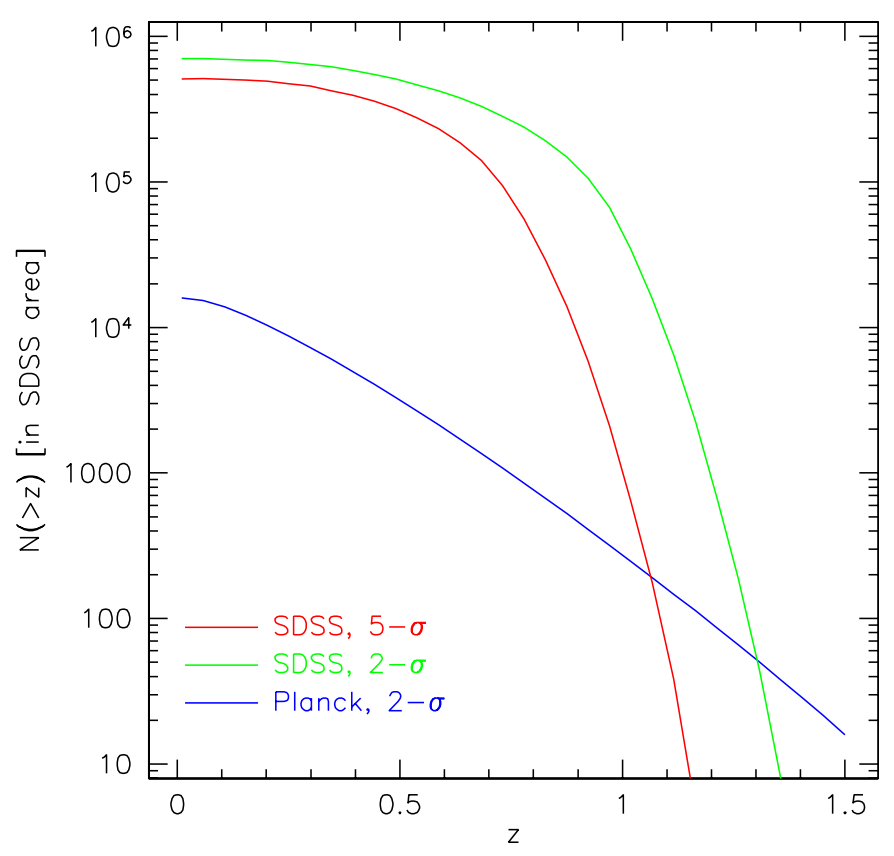

Fig. 10. Cumulative redshift distributions $N(>z)$ of clusters with $M \geq 5 \times 10^{13} h^{-1} M_{\odot}$ in summed $r^{\prime}+i^{\prime}+z^{\prime}$ SDSS data, detected at 2- $\sigma$ and 5- $\sigma$ significance as indicated in the plot. Also shown is the cumulative redshift distribution of clusters detected through the thermal Sunyaev-Zel'dovich effect at 2- $\sigma$ significance by the Planck satellite. Shown is the expected number of clusters in the SDSS area. The abundance of SDSS clusters lies almost two orders of magnitude above that for Planck clusters up to the redshift where clusters with $M=5 \times 10^{13} h^{-1} M_{\odot}$ drop below the detection limit. The SDSS abundances then cut off exponentially. All Planck clusters in the survey area are detected by SDSS out to $z \sim 1.1$ at 5 - $\sigma$, and out to $z \sim 1.3$ at $2-\sigma$. Cluster catalogues constructed from the SDSS data by more traditional techniques are expected to be complete out to redshift $z \sim 0.4$ (Kim et al. 2001), and so should contain about $20 \%$ of the clusters in these deeper catalogues.

which agrees reasonably well with our direct estimates. We thus confirm the speculation by Gonzalez et al. (2001) that the SDSS redshift limit for massive clusters could reach $\sim 1.25$.

We note that it should be possible to estimate redshifts for a large fraction of the SDSS cluster sample through the magnitudes of the brightest cluster galaxies (BCGs). They form a remarkably homogeneous class of objects (Hoessel et al. 1980; Schneider et al. 1983; Postman \& Lauer 1995) with a very narrow luminosity function. Their very narrow colour distribution (Postman \& Lauer 1995; Eisenstein et al. 2001) should greatly simplify their identification even in presence of neighbouring galaxies either in the same cluster or projected on top of it. The $K$ band absolute magnitudes of BCGs in the redshift range $0 \leq z \leq 1$ are compatible with no luminosity evolution, and their colour evolution is consistent with a passively evolving, old stellar population (Aragón-Salamanca et al. 1998). Assuming a reduced absolute magnitude of 21.1 in the $g^{\prime}$ band (cf. Schneider et al. 1983) and a non-evolving 
early-type spectral energy distribution, we estimate the apparent BCG magnitudes shown as functions of redshift in Fig. 9. We checked and confirmed that they are compatible with the $K$-band measurements by AragónSalamanca et al. (1998)

If data in the $g^{\prime}, r^{\prime}$ and $i^{\prime}$ data are summed, it should be possible to identify BCGs out to redshifts near unity, assuming a conservative detection limit of 22.5 in the summed bands. Note that the estimated $r^{\prime}$ magnitudes out to redshifts of $\sim 0.5$ agree very well with the $r^{\prime}$ magnitudes of galaxies with measured redshifts in the SDSS Luminous Red Galaxy Sample (Eisenstein et al. 2001).

Upcoming wide-area surveys in the sub-millimetre regime, like that planned with the Planck satellite (Bersanelli et al. 1996), will allow the detection of galaxy clusters through their peculiar spectral signature caused by the thermal Sunyaev-Zel'dovich effect. Taking the projected temperature sensitivity of Planck and its characteristic beam width of $5^{\prime}$ as a baseline and modelling the cluster population with standard assumptions, Bartelmann (2001) estimated the upper redshift limit of the cluster sample expected to be detectable for Planck. Cumulative redshift distributions for clusters with $M \geq$ $5 \times 10^{13} h^{-1} M_{\odot}$ detected in SDSS and Planck data are shown in Fig. 10.

As explained in Bartelmann (2001), Planck is expected to see clusters at any redshift provided the cluster mass exceeds $\sim 5 \times 10^{14} h^{-1} M_{\odot}$. Below that mass threshold, the cluster population expected to be detectable for Planck in the SDSS survey area is completely contained in the SDSS cluster sample. Since the cluster population is expected to die off rapidly at redshifts beyond unity even in low-density cosmologies, it should be possible to identify almost all ( $\gtrsim 98 \%)$ Planck clusters in the SDSS area with previously detected SDSS clusters. Thus SDSS will provide positions, approximate redshifts and optical luminosities for almost all clusters Planck will see over more than a third of the usable high-latitude sky. Planck is expected to detect of order 1.5 galaxy clusters per square degree at the $2-\sigma$ significance level. Therefore, it should be possible to identify $\sim 15000$ clusters in the SDSS data which are also detectable for Planck, and approximately $70 \%$ of those or $\sim 11000$ clusters will be efficient weak lenses (Bartelmann 2001). The combined data will allow detailed, multi-wavelength studies of a rich, uniquely and homogeneously selected galaxy cluster sample. Correlation of the two data sets (e.g. requiring detection at $\geq 2-\sigma$ in both or stacking Planck data at the positions of SDSS clusters) will allow one to go much deeper (cf. Fig. 10). Note that the Planck clusters which are not visible in SDSS should almost all be at $z \gtrsim 1.2$, so that SDSS offers a way to identify the small high redshift tail of the Planck cluster distribution.

\section{Summary and conclusions}

Dalcanton (1996) suggested searching for galaxy clusters in optical surveys by searching for excess surface bright- ness in heavily smoothed images. A recent application of this technique, the Las Campanas Distant Cluster Survey (Gonzalez et al. 2001) resulted in a catalog of 1073 groups and clusters with redshifts out to $\sim 0.8$.

Prior to smoothing, individually detectable galaxies are removed from the data. Galaxy clusters at sufficiently low redshift $(z \lesssim 0.4)$ can be directly detected as numberdensity enhancements in galaxy counts (Kim et al. 2001). We thus suggest applying Dalcanton's method for finding galaxy clusters beyond that redshift limit. With the individually detectable galaxies removed, the noise in the smoothed data is dominated by Poisson fluctuations in the sky brightness, as the variance due to Poisson and correlated fluctuations in the distribution of undetected galaxies is about an order of magnitude smaller than the variance of the sky brightness.

Drift-scan surveys like the LCDCS are ideal for this type of project as they naturally yield highly uniform images. We investigated in this paper redshift limits for cluster detection in the Sloan Digital Sky Survey data. We assume that

- clusters have surface brightness profiles following the density profile suggested by Navarro et al. (1996, 1997).

- The Universe is well described by a $\Lambda$ CDM model $\left(\Omega_{0}=0.3, \Omega_{\Lambda}=0.7, h=0.7\right)$ normalised to the present number density of galaxy clusters.

- The spectral energy distribution of clusters is a superposition of a dominant early-type and a late-type galaxy spectrum.

- The early-type fraction of galaxy clusters decreases with increasing redshift, reaching half of its present value by redshift unity.

- Early-type galaxies brighten with redshift; and

- the mass-to-light ratio of galaxy clusters at present is $250 M_{\odot} / L_{\odot}$ in the Johnson $B$ band.

Then, using a measurement of the sky brightness at the SDSS telescope site, applying the SDSS photometric system and smoothing with a Gaussian kernel, we showed that:

- The most efficient single SDSS band for galaxy-cluster detection is the $i^{\prime}$ band, in which the $5-\sigma$ detection limit for clusters with mass $M \sim 5 \times 10^{14} h^{-1} M_{\odot}$ is approximately unity.

- Summing data from different bands takes one considerably further. While the $u^{\prime}$ and $g^{\prime}$ bands are not very efficient for this purpose, summed $r^{\prime}+i^{\prime}+z^{\prime}$ data allow clusters with $M \sim 5 \times 10^{14} h^{-1} M_{\odot}$ to be detected at 5 - $\sigma$ significance out to redshift $\sim 1$.

- The limits derived here are in good agreement with extrapolations from the Las Campanas Distant Cluster Survey.

- Brightest cluster galaxies should be detectable in the SDSS data out to redshifts near unity, and their narrow colour distribution should allow them to be distinguished from foreground objects. For a substantial 
fraction of the SDSS cluster sample, it will therefore be possible to derive redshift estimates from the photometry of the brightest cluster members associated with them.

Finally, it is worth noting that, except for the most massive clusters, the SDSS cluster detection redshift limit falls above the upper redshift limit for cluster detections in the sub-millimetre regime expected with the upcoming Planck satellite. This implies that an SDSS cluster sample should contain almost all the clusters Planck is expected to see in the SDSS area, and that it will thus become possible to study a sample of more than $10^{4}$ galaxy clusters in both the optical and sub-millimetre regimes, most of which will be efficient weak gravitational lenses.

Acknowledgements. We wish to thank Stéphane Charlot for providing us with spectral energy distributions, and Michael Strauss for updating the SDSS filter transmission curves.

\section{References}

Aragón-Salamanca, A., Baugh, C. M., \& Kauffmann, G. 1998, MNRAS, 297, 427

Bahcall, N. A., Lubin, L. M., \& Dorman, V. 1995, ApJ, 447, L81

Bahcall, N. A., Cen, R., Davé, R., Ostriker, J. P., \& Yu, Q. 2000, ApJ, 541, 1

Bartelmann, M. 2001, A\&A, 370, 754

Bersanelli, M., et al., Report on Phase A Study for COBRAS/SAMBA, European Space Agency, Paris

Brainerd, T. G., Smail, I., \& Mould, J. 1995, MNRAS, 275, 781

Carlberg, R. G., Yee, H. K. C., Ellingson, E., et al. 1996, ApJ, 462,32
Carlberg, R. G., Yee, H. K. C., \& Ellingson, E. 1997a, ApJ, 478, 462

Carlberg, R. G., Yee, H. K. C., Ellingson, E., et al. 1997b, ApJ, 485, L13

Dalcanton, J. J. 1996, ApJ, 466, 92

van Dokkum, P. G., Franx, M., Kelson, D. D., \& Illingworth, G. D. 1998, ApJ, 504, L17

van Dokkum, P. G., Franx, M., Fabricant, D., Illingworth, G. D., \& Kelson, D. D. 2000, ApJ, 541, 95

Eisenstein, D. J., Annis, J., Gunn, J. E., et al. 2001, AJ, in press; preprint [astro-ph/0108153]

Fukugita, M., Ichikawa, T., Gunn, J. E., et al. 1996, AJ, 111, 1748

Gonzalez, A. H., Zabludoff, A. I., Zaritsky, D., \& Dalcanton, J. J. 2000, ApJ, 536, 561

Gonzalez, A. H., Zaritsky, D., Dalcanton, J. J., \& Nelson, A. 2001, ApJS, in press, preprint [astro-ph/0106055]

Hoessel, J. G., Gunn, J. E., \& Thuan, T. X. 1980, ApJ, 241, 486

Kim, R. S., Kepner, J. V., Postman, M., \& Strauss, M. A. 2001, AJ, in press; preprint [astro-ph/0110259]

Metcalfe, N., Shanks, T., Campos, A., McCracken, H. J., \& Fong, R. 2001, MNRAS, 323, 795

Navarro, J. F., Frenk, C. S., \& White, S. D. M. 1996, ApJ, 462, 563

Navarro, J. F., Frenk, C. S., \& White, S. D. M. 1997, ApJ, 490, 493

Postman, M., \& Lauer, T. R. 1995, ApJ, 440, 28

Postman, M., Lauer, T. R., Szapudi, I., \& Oegerle, E. 1998, ApJ, 506, 33

Schneider, D. P., Gunn, J. E., \& Hoessel, J. G. 1983, ApJ, 264, 337

Woods, D., \& Fahlman, G. G. 1997, ApJ, 490, 11

York, D. G., \& The SDSS Collaboration 2000, AJ, 120, 1579

Zaritsky, D., Shectman, S. A., \& Bredthauer, G. 1996, PASP, 104, 108

Zaritsky, D., Nelson, A. E., Dalcanton, J. J., \& Gonzalez, A. H. 1997, ApJ, 480, L91 